\title{
Dynamics of Domain Walls in the Antiferromagnetic Phase of Dysprosium: a Brief Review
}

\author{
Iu. Liubimova ${ }^{1}$ and S. Kustov ${ }^{2}$ \\ ${ }^{1}$ School of Photonics, ITMO University, Kronverkskiy pr., 49, lit. A, St. Petersburg, 197101, Russia \\ ${ }^{2}$ Department of Physics, University of Balearic Islands, Cra. Valldemossa km 7.5, Palma de Mallorca E07122, Spain
}

Received: June 18, 2020

\begin{abstract}
A brief analysis of several effects related to dynamics of domain walls in antiferromagnetic dysprosium is given. We review experimental studies and interpretations of thermal hysteresis in a number of physical properties of dysprosium and other rare earths that emerges during thermal cycling of samples, scaling laws of magnetic hysteresis, nonequilibrium state of domain walls and relaxation processes in antiferromagnetic phase of dysprosium.
\end{abstract}

\begin{abstract}
Almost all applied properties of ferroics and multiferroics are due to domain walls (DWs). Detailed studies of new features of DWs and the search for their new unusual properties are subjects of "domain wall engineering" [1]: the science of the principles and ways of creating regular stable domain structures for various applications, like information storage. Domain wall engineering deals also with the unusual intrinsic properties of DWs, like superconductivity of twin boundaries in non-stoichiometric tungsten trioxide $\left(\mathrm{WO}_{3}\right)$ [2], magnetoelectric coupling in holmium manganite $\left(\mathrm{HoMnO}_{3}\right)$ [3], electric conductivity of DWs in lithium niobate $\left(\mathrm{LiNbO}_{3}\right)$ [4]. In most cases, the unusual properties of DWs stem from their interaction with other lattice defects, e.g. pinning by point-like defects. Examples of such interactions are reviewed by Salje and Dahmen [5].

In recent years, the properties of antiferromagnets have attracted much attention due to their unusual DW dynamics [5]. Devices based on the antiferromagnets can be used for different applications such as: reading, writing, storing and transmitting data [7-11]. The energy of the antiferromagnetic DWs is
\end{abstract}

often lower than domain wall energy of other ferroics [12], enabling creation of a higher DW density. According to theoretical estimates, the domain wall switching rate is an order of magnitude higher in antiferromagnets than in ferromagnets $[8,9]$. The study of the dynamics of domain walls in antiferromagnetic materials is a difficult experimental task [13]. It requires highly sensitive methods since the motion of an antiferromagnetic domain wall does not change the net magnetic flux across the sample.

Rare-earth elements and their alloys are a particular family of magnetically ordered materials with localized magnetic moments and the highest values of magnetic moment per atom. Dysprosium, as a representative of rare-earth elements, has a complicated temperature-magnetic field phase diagram, shown in Fig. 1, with several ferromagnetic (collinear [14,15], angular [16]) and antiferromagnetic (vortex [17,18], fan [19,20]) spin structures. Rare earths and their alloys are used in innovative research and practical technologies in metallurgy, nuclear energy, optics, medicine, chemical and glass industry, telecommunications 


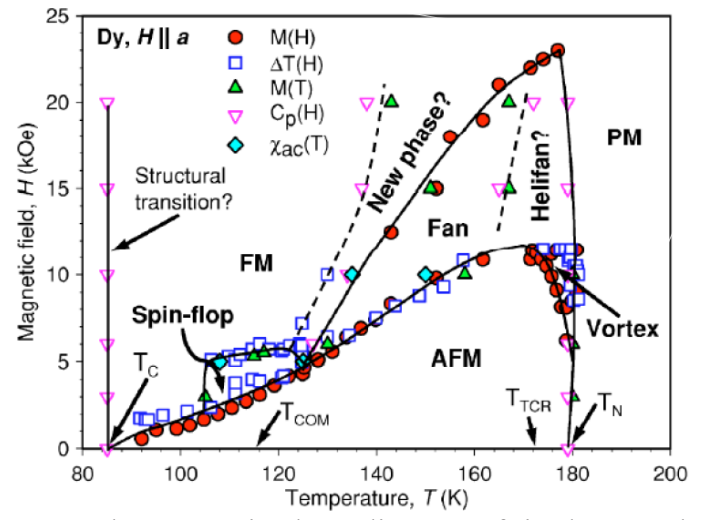

Fig. 1. The magnetic phase diagram of single crystal of dysprosium. At least the following phases are present in the temperature range 200 - 80: ferromagnetic, antiferromagnetic, fan, vortex, paramagnetic. Reproduced from A. S. Chernyshov et al. [17], with permission. Copyright (2005), American Physical Society, DOI: 10.1103/PhysRevB.71.184410.

equipment manufacturing, electronics, laser technology and other applications [6-10,21]. Thus, on one hand, Dy represents a challenging model system for studying magnetically ordered states. On the other hand, despite the complexity of magnetic states, Dy is an important material for various applications.

Under zero magnetic field Dy has a helical antiferromagnetic structure below the Neel temperature $T_{N} \approx 178 \mathrm{~K}[22,23]$. In the helical structure, which results from a competition between positive and negative exchange interactions [24], magnetic moments are confined in the closed packed basal planes of the hexagonal structure, rotating with respect to the $c$-axis. The first-order magnetostructural antiferromagnetic - ferromagnetic transition occurs in Dy at the Curie temperature, $T_{C}=86 \mathrm{~K}$ on cooling, and at $92 \mathrm{~K}$ on heating $[22,23]$. On cooling from $T_{N}$ to $T_{C}$, the rotation angle between spins in the neigh-boring basal planes decreases from $43.2^{\circ}$ to $26.5^{\circ}[23,25]$.

Thermal and magnetic hysteresis are DW-related phenomena in the antiferromagnetic Dy, other rare earths and their alloys. The former is the difference between magnetic, elastic, anelastic and other properties in the antiferromagnetic phase, measured during cooling and subsequent heating. The thermal hysteresis is especially intense after cooling down to the ferromagnetic state [26-29]. The origin of thermal hysteresis was the subject of intense studies based on a variety of experimental methods. Two types of DWs, represented in Fig. 2, are considered in spiral antiferromagnetic structure [28]. The Type I DWs are perpendicular while the Type II DWs are parallel to the $c$-axis, as is shown schematically in Figs. $2 \mathrm{a}$ and $2 \mathrm{~b}$, respectively. Two domains separated by Type I DW have different directions
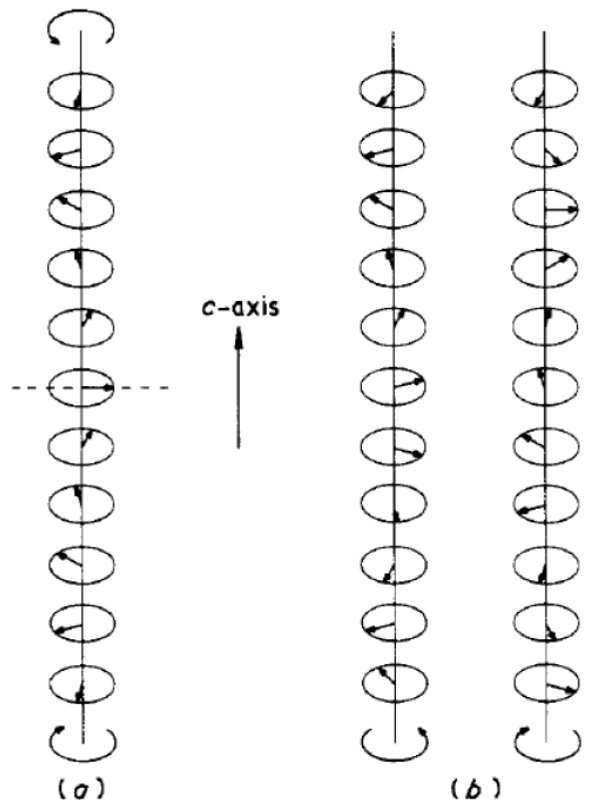

(b)

Fig. 2. Spiral domain walls (a) I type, (b) II type. Reproduced from S. B. Palmer [26], with permission. Copyright (1975), IOP Publishing, DOI: https://doi.org/10.1088/0305-4608/5/12/022.

of spin rotation. Type I DWs possess thus a net magnetic moment, responsible for their interactions. The Type II DW runs parallel to the $c$-axis, and the spin rotation also changes from one domain to another. The structure of Type II DW is rather complex.

Initial experimental studies detected thermal hysteresis only after cooling spiral spin antiferromagnets below $T_{C}$. Del Moral and Lee [26] assumed that the difference between the temperature spectra of magnetic susceptibility during heating and cooling could be the result of the presence, during heating, of "ferromagnetic" DWs separating antiferromagnetic domains. Similar ideas were employed by Palmer and Lee [27] and Palmer [28]. Palmer [28] suggested that the observed anomalous thermal hysteresis of the elastic and anelastic properties of Dy and other rare-earth alloys was due to spiral spin domains. It was assumed that when the sample is heated from temperature below $T_{C}$, the domains of the antiferromagnetic phase "nucleate" from the domains that existed in the ferromagnetic phase. At the Curie temperature the ferromagnetic DWs expand, forming the antiferromagnetic phase. Multiplicity of ferromagnetic domains results in the formation of the antiferromagnetic domains and DWs upon further heating above $T_{C}$. This assumption was apparently consistent with experiment: elastic anomalies were detected only when dysprosium was heated from temperatures below $T_{C}$. Thus, initial works [26-28] attributed thermal hysteresis in rare earths and their alloys to Type I DWs. 
Temperature modulation experiments [30,31] showed that during heating from the ferromagnetic phase thermal hysteresis exists in polycrystalline Dy up to ca. $170 \mathrm{~K}$. This temperature was consistent with the observations of a small effect in the real part of the AC susceptibility at a frequency of $1 \mathrm{kHz}$, cooling-heating rate about $0.3 \mathrm{~K} / \mathrm{min}$ and a magnetic field $H=600 \mathrm{~A} / \mathrm{m}$. The authors supposed that an intermediate domain structure existed in Dy during heating from the ferromagnetic state. Thus, the existence of different antiferromagnetic domain structure during heating from below $T_{C}$ and cooling from above $T_{N}$ was assumed [31]. This assumption was consistent with the conclusions of Palmer [28].

Corró et al. [32] studied both thermal and magnetic hysteresis of the reversible inverse magnetostriction in polycrystalline Dy using so called mechanomagnetic spectroscopy (MMS) [33]. MMS employs ultrasonic resonant oscillations of a sample and detects periodic stress-induced flux variations across the sample. MMS yields reversible inverse magnetostriction (reversible Villari effect) in magnetically ordered materials as a function of oscillatory strain amplitude, magnetic field, and temperature. Under cyclic magnetic field, following [34], MMS yields magnetization $M(H)$ hysteresis and is much more sensitive as compared with conventional measurements of $B(H)$ hysteresis in antiferromagnetic materials (with low susceptibility values). Corró et al. reported a disappearance of magnetoelastic coupling in antiferromagnetic polycrystalline Dy under low magnetic fields at $166 \mathrm{~K}$. This temperature thus represented the socalled Villari point [32]. Quite remarkable was the observation that thermal hysteresis during heating from the ferromagnetic state disappeared in the Villari point. The authors suggested that the thermal hysteresis was due to the presence of a residual ferromagnetic phase, presumably stabilized by lattice defects, like dislocations, creating high local stresses/ strains. The residual ferromagnetic phase existed only up to the temperature of the Villari point, where the magnetoelastic coupling changed the sign. The crucial role of the Villari point was independently confirmed by measurements of real and imaginary components of magnetic susceptibility, Fig. 3 [34].

Dynamics of ferromagnetic DWs is a classical subject of experimental studies [35]. The magnetization of ferromagnets under a slowly growing magnetic field $H$ is often the result of a rearrangement of domain structure through the avalanche-like motion of DWs. Scaling of ferromagnetic hysteresis is well documented [36-38]. On the other hand, very little is known on the DW dynamics in antiferromagnets, mostly due to experimental difficulties. Kobayashi [29] studied $B(H)$ magnetic hysteresis and its scaling

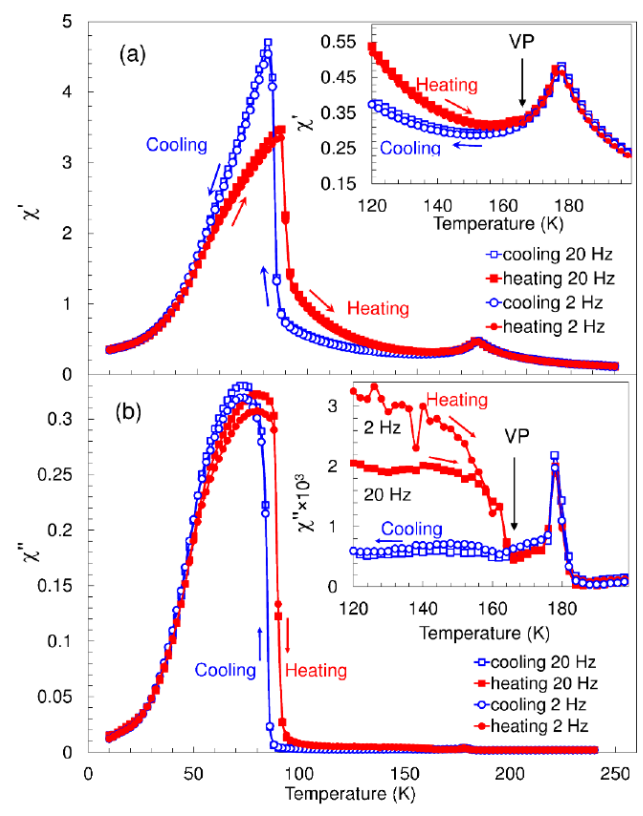

Fig. 3. AC magnetic susceptibility on cooling and heating in the temperature range $9-240 \mathrm{~K}$ : (a) real part $\chi^{\prime}$ and (b) imaginary part $\chi^{\prime \prime}$. Excitation field $3.5 \mathrm{Oe}$ and frequencies $20 \mathrm{~Hz}$ and $2 \mathrm{~Hz}$. The insets show components $\chi^{\prime}$ and $\chi^{\prime \prime}$ on an expanded scale in the range $120-200 \mathrm{~K}$. Reproduced from Iu. Liubimova et al. [31], permission provided by Creative Commons Attribution License. Copyright (2017), Multidisciplinary Digital Publishing Institute, DOI: https://doi.org/10.3390/met7060215.

both in the ferromagnetic and in the helicoidal antiferromagnetic phase of polycrystalline Dy at low fields up to $20 \mathrm{kA} / \mathrm{m}$. Due to resolution limitations, magnetic hysteresis was detected only during heating from the ferromagnetic state and only up to ca. $130 \mathrm{~K}$. Experimental hysteresis loops registered by Kobayashi [29] are shown in Fig. 4. The hysteresis above ca. $130 \mathrm{~K}$ was not resolved and the temperature range with discovered Villari point [32] has not been explored. The temperature range of coexistence of the ferromagnetic and antiferromagnetic phases in polycrystalline Dy was assumed to be $T_{C} \pm 10 \mathrm{~K}$. The magnetic hysteresis in the antiferromagnetic state was explained by the existence of Type I domain walls [29]. Kobayashi [29] attempted to study scaling of antiferromagnetic hysteresis. The dependence of hysteresis losses $\Delta W_{\text {loss }}$ versus remanent magnetic flux density $B_{R}$ is shown in Fig. 5 [29]. Experimental data show power-law scaling $\Delta W_{\text {loss }} \sim\left(B_{R}\right)^{\mathrm{n}}$ with the same exponent $n=1.5$ for antiferromagnetic and ferromagnetic phases. Kobayashi attributed universality of the $\Delta W_{\text {loss }}\left(B_{R}\right)$ scaling to the similarity of the dissipative properties of the motion of DWs in ferro- and antiferromagnetic states. 


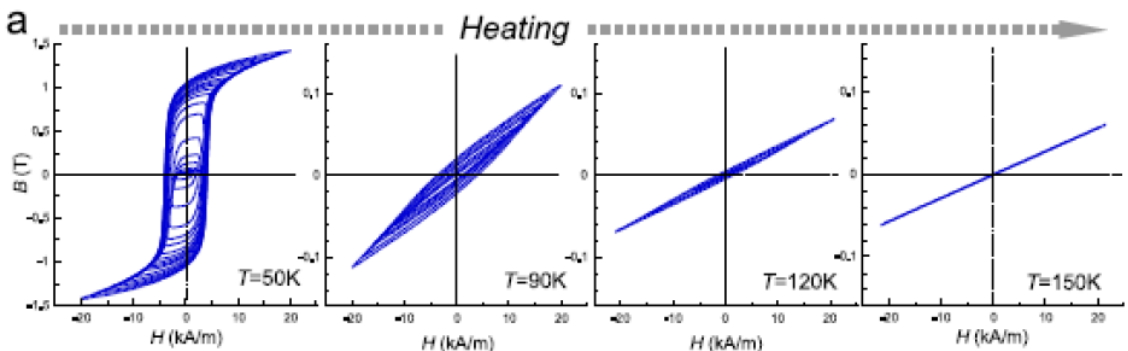

b

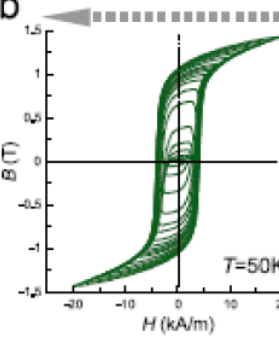

Cooling

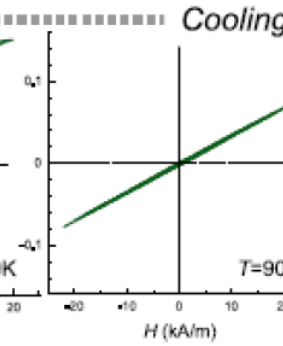

C

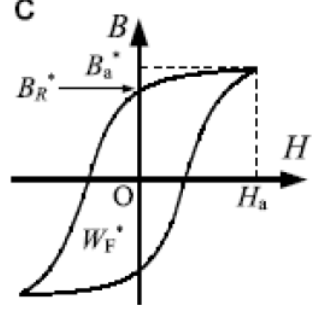

Fig. 4. $B$ - $H$ hysteresis loops with different amplitudes of the magnetic field $H$, shown at different temperatures during (a) heating, (b) cooling; (c) the hysteresis loop parameters $B_{a}^{*}, B_{R}^{*}$ and $W_{F}^{*}$ are the maximum flux density, remanent flux density and hysteresis losses, respectively. Reproduced from S. Kobayashi [29], with permission. Copyright (2011), American Physical Society, DOI: 10.1103/PhysRevLett.106.057207.
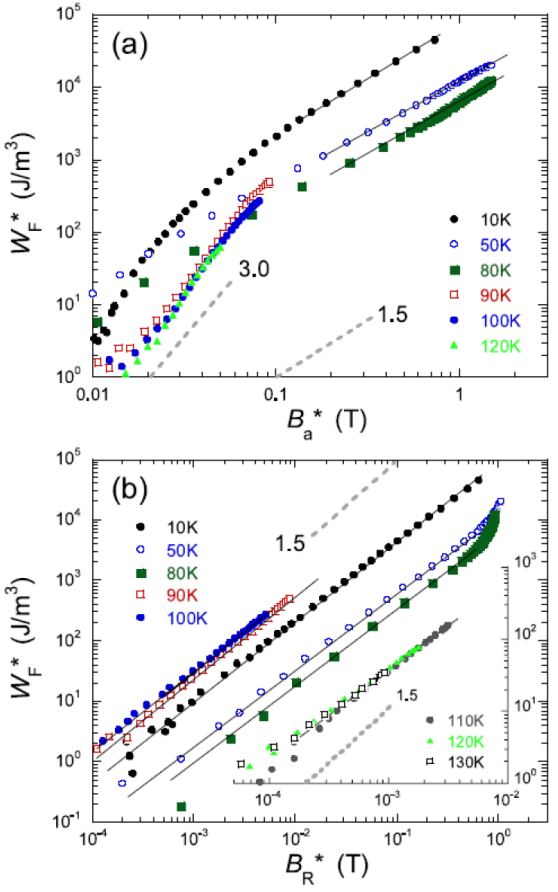

Fig. 5. Relationship between (a) hysteresis losses $W_{F}^{*}$ and maximum magnetic flux density $B_{a}^{*}$; (b) between the hysteretic losses $W_{F}^{*}$ and the remanent magnetic flux density $B_{R}^{*^{*}}$ at different temperatures during heating, plotted on a double logarithmic scale for a polycrystalline dysprosium sample. Reproduced from S. Kobayashi [29], with permission. Copyright (2011), American Physical Society, DOI: 10.1103/PhysRevLett.106.057207.
Chen et al. [39] used resonant magnetic X-ray photon correlation spectroscopy to detect DW fluctuations in $500 \mathrm{~nm}$ epitaxial dysprosium layers (yttrium/dysprosium/yttrium, 20/500/20 nm). Based on autocorrelation of speckle images, exemplified by Fig. 6, the authors argued that i) DWs underwent prolonged fluctuations in the narrow temperature range below the Neel temperature $T_{N}=180 \mathrm{~K}$; ii) the fluctuations of the domain structure froze at temperatures approximately $10 \mathrm{~K}$ below $T_{N}$. It has to be emphasized that the supposed "freezing" temperature is close to the temperature where the thermal hysteresis disappears in Dy. Experimental studies of the ultrafast magneto-optical Kerr effect for the same $500 \mathrm{~nm}$ epitaxial dysprosium layer in the $\mathrm{Y} / \mathrm{Dy} / \mathrm{Y}$ system were reported by Langner et al. [40]. The temperature dependence of the magnetooptical Kerr angle showed a peculiarity close to 166 $\mathrm{K}$, not commented upon.

Elastic and anelastic properties of Dy were studied mostly at low frequencies below ca. $10^{3} \mathrm{~Hz}$ [41-43] and by means of a pulse-echo technique in the $\mathrm{MHz}$ frequency range $[15,25,27,28,44,45]$. Palmer and Lee [27] measured the elastic constants of Dy between 4.2 and $300 \mathrm{~K}$. Their data also pointed to a peculiarity of the $C_{13}$ elastic constant close to the Villari point at $166 \mathrm{~K}$, which remained unexplained. The most informative frequency range for acoustic studies of the DW 
(a)

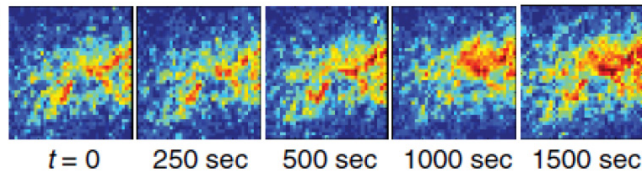

(b)

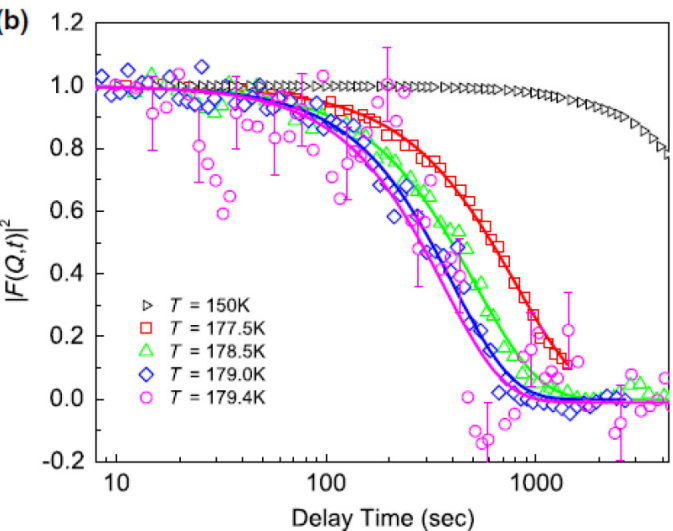

Fig. 6. (a) Snapshots of speckles versus time obtained using X-ray photon correlation spectroscopy at $178.5 \mathrm{~K}$ for a thin film Y/Dy/Y; (b) normalized autocorrelation function at various temperatures, the rate of change of function as it approaches Neel's temperature becomes higher. Reproduced from S. W. Chen et al. [39], with permission. Copyright (2013), American Physical Society, DOI: 10.1103/PhysRevLett.110.217201.

dynamics in ferromagnets is usually between $10^{3}$ and $10^{5} \mathrm{~Hz}$. These frequencies are high enough to make detectable the microeddy current component, proportional to the frequency, and do not exceed strongly the relaxation frequency of macroeddy current component, see e.g. [46]. Frequencies from this range were used to study elastic and anelastic properties in the antiferromagnetic state of polycrystalline Dy $[47,48]$. Resonant longitudinal oscillations of barshaped samples at a frequency around $90 \mathrm{kHz}$ were employed. Fig. 7 shows an example of temperature spectra of ultrasonic internal friction (IF), expressed as logarithmic decrement $\delta$, and Young's modulus [48]. The IF is extremely low in the paramagnetic state $\left(\delta \approx 10^{-5}\right)$ and increases nearly an order of magnitude upon antiferromagnetic ordering. Temperature hysteresis is clearly detected in elastic and anelastic properties even during thermal cycling within antiferromagnetic phase. These two observations pointed to the predominant contribution of DWs to anelasticity of antiferromagnetic Dy [48]. Interrupted cooling and heating scans exemplified in Fig. 7c, revealed intense IF relaxation and T-dot dependences $[47,48]$ with a maximum intensity located at the Villari point, $T_{V P}=166 \mathrm{~K}$, marked also by an IF peak. A conclusion was drawn that antiferromagnetic DWs in polycrystalline Dy are in the non-equilibrium "glassy" state down to ca. $150 \mathrm{~K}$, characterized also by memory and temperature chaos effects [48].
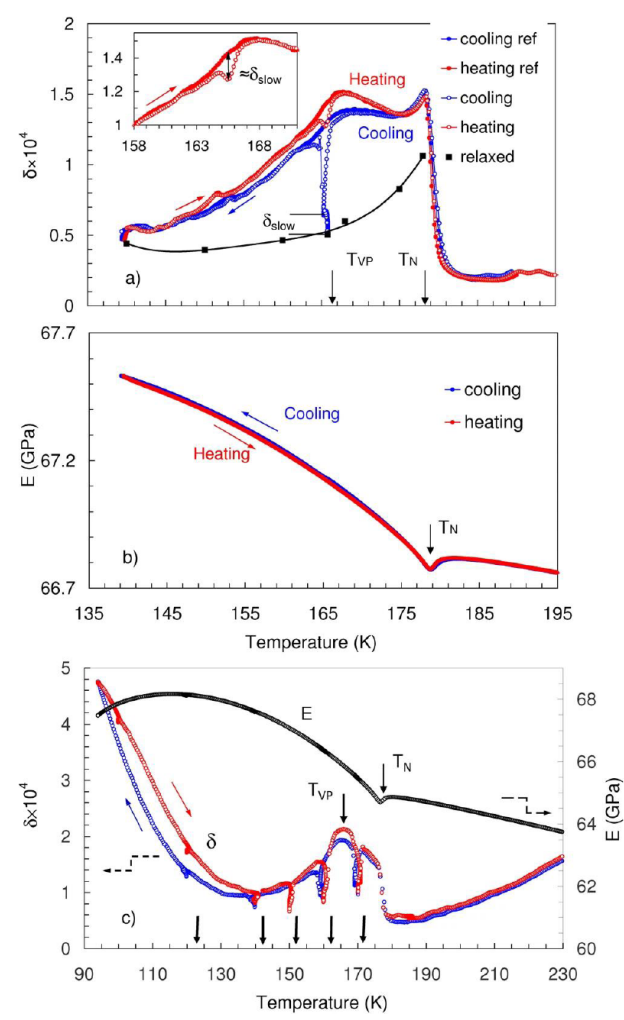

Fig. 7. (a) Temperature spectra of internal friction, $\delta$, in two consecutive thermal cycles $210 \mathrm{~K}-140 \mathrm{~K}$, cooling-heating rate $-1 \mathrm{~K} / \mathrm{min}$. The first cooling process was interrupted at $166 \mathrm{~K}$ for $2400 \mathrm{~s}$, resulting in a significant relaxation of the internal friction. The reference cooling-heating curves correspond to the uninterrupted thermal cycle. Black squares represent final values of the internal friction after interrupting cooling scan for $2400 \mathrm{~s}$ at fixed temperatures: $140,150,160,166,168,175$, and $178 \mathrm{~K}$. The inset shows details of the memory effect on heating. (b) Temperature spectra of Young's modulus during thermocycling for as cast sample. (c) Dependencies of internal friction and Young's modulus, E, in a thermocycle $230-95 \mathrm{~K}$ (coolingheating rate $2 \mathrm{~K} / \mathrm{min}$ ) with interruptions of thermal cycling for as received (rolled) sample. Reproduced from S. Kustov et al. [48], permission provided by Creative Commons Attribution 4.0 International License, http:/creativecommons.org/licensesby/4.0/. Copyright (2019), Nature Publishing Group, DOI: https://doi.org/10.1038/s41598-019-41566-7.

Direct observations of intense anelastic relaxations $[47,48]$ and frequency dependence of imaginary part of susceptibility [34] contradicts observations of DW stability by means of X-ray photon correlation spectroscopy in thin epitaxial films [39]. Kustov et al. [48] concluded that DW fluctuations just below the Néel temperature reported in [39], reflect DW fluctuations during the second order 
para-antiferromagnetic phase transition. The IF relaxation in the antiferromagnetic polycrystalline dysprosium down to $150 \mathrm{~K}$ showed two kinetic processes: "fast" and "slow" [48]: The former scales with the temperature rate, T-dot, and was attributed to the rapid rearrangement of the DW structure similar to the so-called transitory damping term. The latter showed logarithmic kinetics, typical in glassy structures. Only the slow relaxation component was found to be associated with the memory effect [48], a hallmark of spin glasses.

\section{CONCLUSIONS}

Experimental results accumulated so far and their interpretations allows one to draw the following conclusions.

The thermal hysteresis that occurs during thermal cycling of Dy, other rare earths and their alloys can be consistently explained by the existence on heating of "extra" Type I DWs, inherited from the ferromagnetic phase or from the ferromagnetic nuclei. The residual ferromagnetic phase can be stabilized by lattice defects up to the Villari point, wherein magnetoelastic coupling in polycrystalline Dy vanishes and changes the sign.

The Villari point around $166 \mathrm{~K}$ in polycrystalline Dy is an important characteristic temperature. This temperature controls the behavior of several magnetic properties as detected by a number of different methods.

The observation of relaxation of the reversible Villari effect and internal friction in the antiferromagnetic state from the Néel temperature down to ca. $150 \mathrm{~K}$, as well as the existence of the "memory" effect, point to the nonequilibrium glassy state of antiferromagnetic domain walls over this temperature range.

\section{REFERENCES}

[1] E.K.H. Salje, Multiferroic domain boundaries as active memory devices: Trajectories toward domain boundary engineering, ChemPhysChem, 2010, vol. 11, no. 5, pp. 940-950, https://doi. org/10.1002/cphc. 200900943

[2] Y. Kim, M. Alexe and E.K.H. Salje, Nanoscale properties of thin twin walls and surface layers in piezoelectric $\mathrm{WO}_{3-x}$, Appl. Phys. Lett., 2010, vol. 96, no. 3, 032904, https://doi.org/10.1063/ 1.3292587

[3] T. Lottermoser and M. Fiebig, Magnetoelectric behavior of domain walls in multiferroic $\mathrm{HoMnO}_{3}$, Phys. Rev. B, 2004, vol. 70, 220407(R), https://doi.org/10.1103 PhysRevB.70.220407

[4] P. Chaudhary, H. Lu, A. Lipatov, Z. Ahmadi, J. Mcconville, A. Sokolov, J. Shield,
A. Sinitskii, J.M. Gregg, and A. Gruverman, Low-Voltage Domain-Wall LiNbO ${ }_{3}$ Memristors, Nanoletters, 2020, XXXX, XXX, XXX-XXX, https://doi.org/10.1021/acs.nanolett.0c01836

[5] E.K.H. Salje and K.A. Dahmen, Crackling Noise in Disordered Materials, Annu. Rev. Condens. Matter Phys., 2014, vol 5, pp. 233 254, https://doi.org/10.1146/annurevconmatphys-031113-133838

[6] A. Fukuda Y. Takanishi, T. Isozaki, K. Ishikawa and H. Takezoe, Antiferroelectric chiral smectic liquid crystals, J. Mater. Chem., 1994, vol. 4, pp. 997-1016, https://doi.org/10.1039/ JM9940400997

[7] B.G. Park, J. Wunderlich, X. Martí, V. Holý, Y. Kurosaki, M. Yamada, H. Yamamoto, A. Nishide, J. Hayakawa, H. Takahashi, A.B. Shick and T. Jungwirth, A spin-valve-like magnetoresistance of an antiferromagnet-based tunnel junction, Natur. Mater., 2011, vol. 10, pp. 347-351, https://doi.org/10.1038/nmat2983

[8] P. Wadley, B. Howells, J. Zelezny, C. Andrews, V. Hills, R.P. Campion, V. Novak, F. Freimuth, Y. Mokrousov, A.W. Rushforth, K.W. Edmonds, B.L. Gallagher and T. Jungwirth, Electrical switching of an antiferromagnet, Science, 2016, vol. 351, no. 6272, pp. 587-590, https://doi.org/ 10.1126/science.aab1031

[9] O. Gomonay, T. Jungwirth and J. Sinova, High Antiferromagnetic Domain Wall Velocity Induced by Néel Spin-Orbit Torques, Phys. Rev. Lett., 2016, vol. 117, 017202, https://doi. org/10.1103/PhysRevLett.117.017202

[10] T. Jungwirth, J. Sinova, A. Manchon, X. Marti, J. Wunderlich and C. Felser, The multiple directions of antiferromagnetic spintronics, Nature Phys., 2018, vol. 14, pp. 200-203, https://doi.org/10.1038/s41567-018-0063-6

[11] R. Duine, Spintronics: an alternating alternative, Nature Mater., 2011, vol. 10, pp. 344-345, https://doi.org/10.1038/nmat3015

[12] D.D. Viehland and E.K.H. Salje, Domain boundary-dominated systems: adaptive structures and functional twin boundaries, Adv. Phys., 2014, vol. 63, no. 4, pp. 267-326, https://doi.org/10.1080/00018732.2014.974304

[13] E.G. Tveten, T. Müller, J. Linder and A. Brataas, Intrinsic magnetization of antiferromagnetic textures, Phys. Rev. B, 2016, vol. 93, 104408, https://doi.org/10.1103/ PhysRevB.93.104408

[14] A.V. Andrianov, A.N. Vasil'ev and Y.P. Gaidukov, Low temperature magnetic phase diagrams of dysprosium and gadolinium, Physica B, 1991, vol. 169, no. 1-4, pp. 469-470, https://doi.org/10.1016/0921 4526(91)90280-R 
[15] A.V. Andrianov, Y.P. Gaidukov, A.N. Vasil'ev and E. Fawcett, The magnetic phase diagrams of dysprosium, J. Magn. Magn. Mater., 1991, vol. 97, no. 1-3, pp. 246-250, https://doi.org/10.1016/0304-8853(91)90188-G

[16] M.T. Alkhafaji and N. Ali, Magnetic phase diagram of dysprosium, J. Alloys Compd., 1997, vol. 250, no. 1-2, pp. 659-661, https:// doi.org/10.1016/S0925-8388(96)03039-3

[17] A.S. Chernyshov, A.O. Tsokol, A.M. Tishin, K.A. Gschneidner and V.K. Pecharsky, Magnetic and magnetocaloric properties and the magnetic phase diagram of singlecrystal dysprosium, Phys. Rev. B, 2005, vol. 71, 184410, https://doi.org/10.1103/ PhysRevB.71.184410

[18] E.B. Amitin, V.G. Bessergenev and Y.A. Kovalevskaya, Features of the thermodynamic properties of dysprosium as a quasi-twodimensional magnetic system, Soviet Phys. JETP, 1983, vol. 57, no. 1, pp. 117-122, http:// jetp.ac.ru/cgi-bin/dn/e_057_01_0117.pdf

[19] C. Isci and S.B. Palmer, An ultrasonic study of the magnetic phases of dysprosium, J. Phys. F: Met. Phys., 1978, vol. 8, no. 2, pp. 247-260, https://doi.org/10.1088/0305- 4608/8/2/011

[20] A.S. Chernyshov, Y. Mudryk, V.K. Pecharsky and K.A. Gschneidner, Temperature and magnetic field-dependent $x$-ray powder diffraction study of dysprosium, Phys. Rev. B, 2008, vol. 77, 094132, https://doi.org/10.1103/ PhysRevB.77.094132

[21] A.R. Jha, Rare-earth materials: properties and applications (Taylor and Francis Group, New York, 2014)

[22] J.F. Elliott, S. Legvold and F.H. Spedding, Some magnetic properties of Dy metal, Phys. Rev., 1954, vol. 94, no. 5, pp. 1143-1145, https://doi.org/10.1103/PhysRev.94.1143

[23] M.K. Wilkinson, W.C. Koehler, E.O. Wollan and J.W. Cable, Neutron diffraction investigation of magnetic ordering in Dysprosium, J. Appl. Phys., 1961, no. 32, p. S48, https://doi.org/10.1063/1.2000493

[24] K.P. Belov, R.Z. Levitin and S.A. Nikitin, Ferromagnetism and antiferromagnetism of rare-earth metals, Sov. Phys. Usp., 1964, vol. 7, no. 2, pp. 179-208, http://dx.doi. org/10.1070/PU1964v007n02ABEH003660

[25] M. Rosen, Elastic Moduli and Ultrasonic Attenuation of Gadolinium, Terbium, Dysprosium, Holmium, and Erbium from 4.2 to $300^{\circ} \mathrm{K}$, Phys. Rev., 1968, vol. 174, no. 2, pp. 504-514, https://doi.org/10.1103/ PhysRev.174.504
[26] A. Del Moral and E.W. Lee, The reversible susceptibilities of dysprosium and terbium, J. Phys. F: Met. Phys., 1974, vol. 4, no. 2, pp. 280-290, https://doi.org/10.1088/03054608/4/2/015

[27] S.B. Palmer and E.W. Lee, The Elastic Constants of Dysprosium and Holmium, Proc. R. Soc. Lond. A, 1972, vol. 327, pp. 519-543, https://doi.org/10.1098/rspa.1972.0061

[28] S.B. Palmer, Antiferromagnetic domains in rare earth metals and alloys, J. Phys. F: Met. Phys., 1975, vol. 5, no. 12, pp. 2370-2378, https://doi.org/10.1088/0305-4608/5/12/022

[29] S. Kobayashi, Universal hysteresis scaling for incommensurate magnetic order in dysprosium,Phys. Rev. Lett., 2011, vol. 106, 057207, https://doi.org/10.1103/PhysRevLett. 106.057207

[30] T.J. Mckenna, S.J. Campbell, D.H. Chaplin and G.V.H. Wilson, Domain effects near the order-disorder and order-order ferromagnetic transitions in Gd and Dy, J. Magn. Magn. Mater., 1980, vol. 20, no. 2, pp. 207-211, https://doi.org/10.1016/0304-8853(80)90458-8

[31] T.J. Mckenna, S.J. Campbell, D.H. Chaplin and G.V.H. Wilson, Temperature Modulation and Temperature Hysteresis Studies of Dysprosium, Phys. Status Solidi A, 1983, vol. 75, no. 2, pp. 421-432, https://doi.org/10.1002/pssa.2210750211

[32] M.L. Corró, A.El Hichou, E. Cesari and S. Kustov, Study of magnetic transitions in Dy by means of reversible Villari effect, J. Phys. D: Appl. Phys., 2016, vol. 49, 015001, https://doi.org/10.1088/0022-3727/49/1/015001

[33] S. Kustov, F. Masdeu and E. Cesari, Mechanomagnetic spectroscopy of phase transitions in ferromagnetic shape memory alloys, Appl. Phys. Lett., 2006, vol. 89, art. 061917, https://doi.org/10.1063/1.2335813

[34] I. Liubimova, M. Corró Moyà, J. Torrens-Serra, V. Recarte, J. Pérez-Landazábal and S. Kustov, Low Field Magnetic and Thermal Hysteresis in Antiferromagnetic Dysprosium, Metals, 2017, vol. 7, no. 6, p. 215, https://doi.org/10.3390/met7060215

[35] G. Bertotti, Hysteresis in magnetism (Academic Press, San Diego, California, USA, 1998).

[36] J.P. Sethna, K.A. Dahmen and C.R. Myers, Crackling noise, Nature, 2001, vol. 410, no., pp. 242-250, https://doi.org/10.1038/35065675

[37] C.P. Steinmetz, On the law of hysteresis, Proc. IEEE, 1984, vol. 72, no. 2, pp. 197-221; https://doi.org/10.1109/PROC.1984.12842 
[38] S. Kobayashi, S. Takahashi, T. Shishido, Y. Kamada and H. Kikuchi, Low-field magnetic characterization of ferromagnets using a minor-loop scaling law, J. Appl. Phys., 2010, vol. 107, art. 023908, https://doi.org/10.1063/1.3289317

[39] S.W. Chen, H. Guo, K.A. Seu, K. Dumesnil, S. Roy and S.K. Sinha, Jamming behavior of domains in a spiral antiferromagnetic system, Phys. Rev. Lett., 2013, vol. 110, 217201, https://doi.org/10.1103/PhysRevLett.110.217201

[40] M.C. Langner, S. Roy, A.F. Kemper, Y.D. Chuang, S.K. Mishra, R.B. Versteeg, Y. Zhu, M.P. Hertlein, T.E. Glover, K. Dumesnil and R.W. Schoenlein, Scattering bottleneck for spin dynamics in metallic helical antiferromagnetic dysprosium, Phys. Rev. B, 2015, vol. 92, 184423, https://doi.org/10.1103/ PhysRevB.92.184423

[41] I.M. Sharshakov, A.N. Korshunov and G.L. Polner, Magnetic transformations and internal friction in polycrystalline dysprosium, Fiz. Met. Metalloved., 1978, vol. 45, no. 1, pp. 204-205.

[42] A.M. Tishin and O.A. Shipilov, Anomalous internal-friction in dysprosium at lowtemperatures, Fiz. Tverd. Tela, 1992, vol. 34, pp. 3554-3556.

[43] G.S. Burkhanov, S.A. Nikitin, A.M. Tishin, O.D. Chistyakov and O.A. Shipilov, A study of elastic phenomena and relaxation processes in Dysprosium, Mosc. Un. Phys. B., 1993, vol. 48, no. 2 , p. 43.

[44] R.A. Treder and M. Levy, Ultrasonic study of dysprosium in a magnetic field, J. Magn. Magn. Mater., 1977, vol. 5, no. 1, pp. $9-17$, https://doi.org/10.1016/0304-8853(77)90191-3

[45] M. Rosen and H. Klimker, Low-Temperature Elasticity and Magneto-Elasticity of Dysprosium Single Crystals, Phys. Rev. B, 1970, no. 1, 3748, https://doi.org/10.1103/ PhysRevB.1.3748

[46] S. Kustov, M.-Ll. Corró, V. Kaminskii, A. Saren and K. Ullakko, Elastic and anelastic phenomena related to eddy currents in cubic $\mathrm{Ni}_{2} \mathrm{MnGa}$, Scr. Mater., 2018, vol. 147, pp. 69-73, https://doi.org/10.1016/j.scriptamat.2018.01.003

[47] Iu. Liubimova, K. Sapozhnikov, V. Nikolaev, M.-Ll. Corró and S. Kustov, Ultrasonic study of thermal hysteresis in helical antiferromagnetic Dy, Rev. Adv. Mater. Sci., 2018, 57 (2018) 241-245, https://doi.org/ 10.1515/rams-2018-0069

[48] S. Kustov, Iu. Liubimova, M. Corró, J. Torrens-Serra, X. Wang, C.R.S. Haines and E.K.H. Salje, Temperature Chaos, Memory Effect, and Domain Fluctuations in the Spiral Antiferromagnet Dy, Sci. Rep., 2019, vol. 9, 5076, https://doi.org/10.1038/ s41598-019- 41566-7 